\title{
Senescent cells and osteoarthritis: a painful connection
}

\author{
Ok Hee Jeon, ${ }^{1,2}$ Nathaniel David, ${ }^{3}$ Judith Campisi, ${ }^{2}$ and Jennifer H. Elisseeff ${ }^{1}$ \\ ${ }^{1}$ Translational Tissue Engineering Center, Wilmer Eye Institute and Department of Biomedical Engineering, Johns Hopkins University, Baltimore, Maryland, USA. 'Buck Institute for Research on Aging, \\ Novato, California, USA. 3̈nity Biotechnology, Brisbane, California, USA.
}

\begin{abstract}
Senescent cells (SnCs) are associated with age-related pathologies. Osteoarthritis is a chronic disease characterized by pain, loss of cartilage, and joint inflammation, and its incidence increases with age. For years, the presence of SnCs in cartilage isolated from patients undergoing total knee artificial implants has been noted, but these cells' relevance to disease was unclear. In this Review, we summarize current knowledge of SnCs in the multiple tissues that constitute the articular joint. New evidence for the causative role of $\mathrm{SnCs}$ in the development of posttraumatic and age-related arthritis is reviewed along with the therapeutic benefit of SnC clearance. As part of their senescence-associated secretory phenotype, SnCs secrete cytokines that impact the immune system and its response to joint tissue trauma. We present concepts of the immune response to tissue trauma as well as the interactions with SnCs and the local tissue environment. Finally, we discuss therapeutic implications of targeting SnCs in treating osteoarthritis.
\end{abstract}

\section{Introduction}

Osteoarthritis (OA), the most common joint disease of late life, affects $80 \%$ of individuals over age 65 (1). Women and obese individuals are more susceptible to this chronic disease (2). Younger patients are also at risk of developing OA after joint trauma (posttraumatic OA [PTOA]). OA manifests as joint pain, swelling, and, ultimately, mechanical failure. While OA affects joints throughout the body, its current understanding comes largely from study of OA of the knee. Multiple tissues within the knee contribute to OA progression (Figure 1). Cartilage lining the surfaces of articulating joints provides a low-friction surface, enabling painless joint movement (3). The entire joint is enclosed by a membranous structure known as the synovial membrane, or synovium, which produces synovial fluid that bathes the joint and serves as both a source of nutrients and a mechanical lubricant. These tissue components communicate and function together to create the joint "organ" and maintain joint function.

While OA is often characterized as gradual cartilage loss by wear and tear, the disease is more complex and includes dysfunction in all the joint components. Cartilage tissue fractures at sites of high mechanical stress, a process termed fibrillation. In these fibrillations, and in cartilage tissue throughout the joint, the extracellular matrix (ECM) loses proteoglycans and collagen fibers. Beyond these changes, the subchondral bone forms osteophytes, fibrocartilage-capped bony outgrowths that are present on the joint margins, and dysfunctional synovial fluid compromises lubrication. Osteophytes are formed by endochondral ossification - bone deposition and formation of bone marrow $(\mathrm{BM})$ cavities. This process starts with fibroblast-like cells

Conflict of interest: JHE, OHJ, ND, and JC are inventors on patents owned by Unity Biotechnology, Buck Institute, Mayo Clinic, and Johns Hopkins and licensed to Unity Biotechnology $(9,849,218$ and $9,855,266)$. JHE is a consultant to, and ND and JC are founders of, Unity Biotechnology.

Reference information: J Clin Invest. 2018;128(4):1229-1237.

https://doi.org/10.1172/JCI95147. in the periosteum differentiating to chondrocytes that deposit cartilage matrix, which serves as the template for new bone (4). Finally, the synovium thickens and is frequently inflamed (5). The menisci are often damaged and can displace into the joint space (6). These changes in tissue structure and function are accompanied by pain and reduced joint mobility that contribute to OA's clinical symptoms.

Participation in high-impact physical activities, including military service and athletics, places younger patients at higher risk of developing PTOA (7-9). Damage or loss of the anterior cruciate ligament (ACL) in the knee, with or without meniscus injury, frequently leads to PTOA (10). In addition to wear and tear on the joints, OA is associated with low-grade systemic and joint inflammation created by proinflammatory and matrixdegrading cytokines (11-13). The pathogenic mechanisms involved in OA progression remain under debate. For example, transcriptome analysis identified a link between inflammation and joint trauma (ACL rupture and surgical destabilization of the medial meniscus, abbreviated DMM) in animal models of PTOA (14). Differential expression of these inflammatory molecules and their related pathways, however, was not found in chondrocytes isolated from normal and human OA cartilage (15, 16). Further, repairing a mechanical injury or instability does not appear to prevent PTOA development (17), suggesting that additional biological mechanisms beyond simple mechanical wear and tear or purely inflammatory factors contribute to the clinical disease. Understanding how trauma and aging contribute to OA development will provide insights into disease mechanisms as well as new therapeutic targets. Particularly in cases of younger patients who retain regenerative capacity, interventions may not only stop OA progression but also lead to the rebuilding of new tissue to restore healthy organ function.

Cellular senescence is potentially a common molecular mechanism that drives or promotes both age-associated OA and PTOA (18-22). Shared molecular features of aging and many forms of stress/trauma include genomic instability (including telomere 


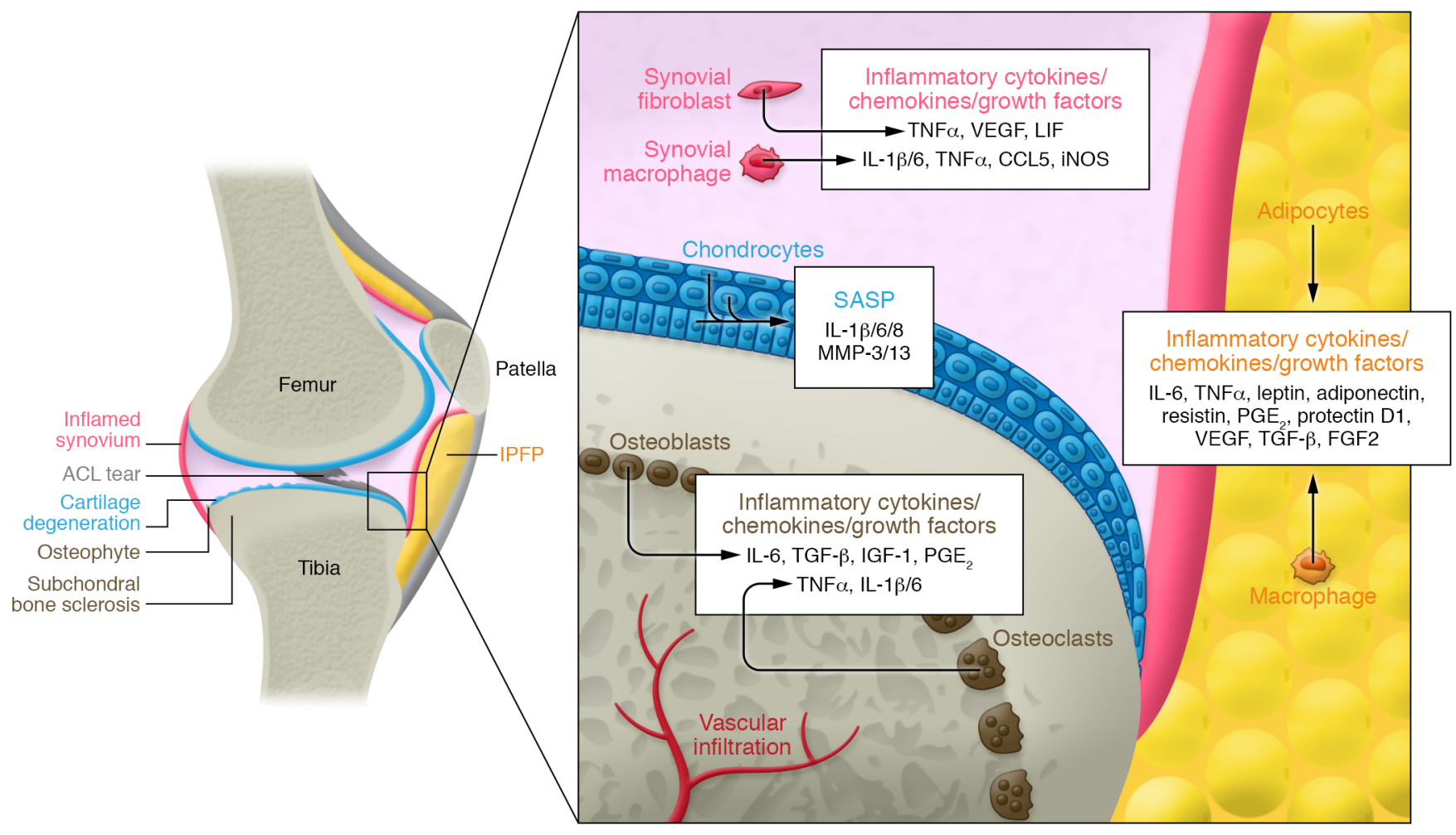

Figure 1. A schematic overview of the joint structure. Articular cartilage, subchondral bone, synovium, and infrapatellar fat pad (IPFP) are joint tissues that may harbor senescent cells and secrete a senescence-associated secretory phenotype (SASP) induced by aging or trauma. Loss or damage to the anterior cruciate ligament $(\mathrm{ACL})$ or other joint structures is a primary cause of PTOA.

attrition and epigenetic alterations), dysregulated nutrient sensing, loss of proteostasis, mitochondrial dysfunction, stem cell exhaustion, and cellular senescence (23). Senescent cells (SnCs) are characterized by their inability to divide as well as resistance to apoptosis and a robust proinflammatory secretome known as the senescence-associated secretory phenotype (SASP) that can alter the structure and function of surrounding cells and tissues (24). Identifying and manipulating SnCs has remained a challenge until recently. Here, we review recent progress in defining the role of SnCs and the SASP in the development of age- and trauma-induced OA. We discuss the presence and impact of SnCs in each joint tissue, the immunological relevance of SnCs, and the impact of the SASP on disease and regeneration. Finally, we will discuss future prospects for eliminating SnCs to treat disease.

\section{Cellular senescence}

Replicative senescence was historically thought to underlie the decline in tissue homeostasis, repair, and regeneration that accompanies aging. Later discoveries identified additional extrinsic and intrinsic stimuli causing cells to undergo senescence, including genomic damage (e.g., telomere attrition, DNA double-strand breaks or other lesions; ref. 25), derangements or damage to the epigenome (26), various oncogenic mutations (e.g., activated Ras) (27), extrinsic or intrinsic oxidative stress (28), and many metabolic changes, particularly those that cause mitochondrial dysfunction (29). Most of these stressors put cells at risk of developing cancer; thus, senescence-associated growth arrest serves as a potent tumor-suppressive mechanism (24).
The senescence response induced by one or more of these stressors generally results from the activation of molecular stress-response pathways. The p53/p21 and/or p16 $6^{\mathrm{INK} 4 \mathrm{~A}} /$ retinoblastoma pathways are the primary drivers of permanent senescence-associated proliferative arrest, although other pathways may also implement a senescence response (30-32). SnCs are transiently induced and play a beneficial role in embryonic development $(33,34)$, wound healing $(35)$, and insulin secretion by pancreatic $\beta$ cells (36). However, as discussed below, chronic presence of SnCs, as observed in aged tissues, is associated with loss of tissue function and age-related chronic diseases such as OA.

Although SnCs are nondividing, their persistence is far from harmless. They can negatively impact surrounding cells and tissues, primarily through their SASP (24, 37-39). The SASP entails secretion of several extracellular proteases, proinflammatory cytokines, chemokines, and growth factors (39). The specific secretory profiles of SnCs are highly cell- and context-dependent and thus define the potential positive or negative impact of SnCs. SnCs may have distinct effects depending on the inducing stressor and the local tissue environment (29, 40-42).

Cell culture models provide some insights into the variability of the SASP composition. For example, oncogene-induced senescent human fetal lung fibroblasts produce 103 distinct proteins (43). Several of these SASP proteins overlap with radiationinduced senescence in the same cells, but the SASPs induced by oncogenic Ras versus genotoxic stress also have distinct features (39). SnCs induced by genotoxic stress and oncogenes elicit a SASP that includes IL-1- and NF- $\mathrm{kB}$-dependent factors (40). SnCs 
induced by mitochondrial dysfunction-associated senescence (MiDAS), which activates the NAD/AMPK/p53 pathway (29), express a SASP that differs from genotoxic- or oncogene-induced SASPs. The MiDAS SASP lacks IL-1- and NF- $\mathrm{BB}-$ dependent proteins but includes the antiinflammatory cytokine IL-10, the proinflammatory chemokines CCL27 and TNF- $\alpha$, and the growth factor amphiregulin (29). Several of the common SASP factors, including IL-1, IL-6, and matrix metalloproteinase 3 (MMP-3), overlap with mediators of OA inflammation. SnCs induced by either stimuli secrete the high-mobility group box 1 (HMGB1) protein, the founding member of a growing class of proinflammatory factors termed alarmins or damage-associated molecular patterns (DAMPs) (42). Alarmins and DAMPs connect the presence of SnCs with tissue damage and repair responses such as those in joint trauma.

\section{SnCs in the knee}

Inflammation and tissue degradation are hallmarks of OA that can be mediated by SASP-associated factors. SnCs and their SASPs are not OA-specific but are needed to generate an inflammatory, degradative tissue microenvironment similar to that found in OA. Several SnC markers, including p16 ${ }^{\mathrm{INK} 4 \mathrm{~A}}$ and p21 expression (44), are found in multiple tissues in arthritic joints, including cartilage, subchondral bone, synovium, and the infrapatellar fat pad (IPFP) $(21,22,45,46)$. However, their role, their tissue-specific SASP, their evolution over time, and ultimately their disease relevance are not yet fully elucidated. Here, we review our current understanding of SnCs in joint tissues.

Cartilage. Articular cartilage is an avascular and aneural tissue composed of a rich ECM comprising primarily type II collagen and aggrecan. The tissue is populated primarily by chondrocytes that are responsible for cartilage maintenance through a balance of ECM synthesis and remodeling by MMPs. In the early phase of OA, chondrocytes near the superficial layer divide, form clusters, and exhibit increased anabolic activity in an attempt to repair the tissue. During disease progression, the chondrocyte phenotype that maintains tissue is lost. Instead the phenotype is skewed toward matrix destruction and inflammatory cytokine production, primarily through collagen-degrading MMP-13 and aggrecandegrading ADAMTS-5 $(11,12)$. The proteoglycans become depleted, the collagen network is eroded, and chondrocytes become hypertrophy-like, leading to calcified cartilage (47).

For many years, senescent chondrocytes were noted in cartilage tissue discards from diseased joints after total knee arthroplasty surgery $(22,48-50)$. Senescent chondrocytes accumulate with age and are present at higher numbers in human OA cartilage compared with age-matched healthy cartilage $(18,21,45,46,50-52)$. The localization of senescent chondrocytes near osteoarthritic lesions, but not in intact tissue, further suggests a disease connection (46). The positive correlation between the presence and degree of senescence and disease severity $(46,49)$, however, remains controversial. Ongoing clinical trials will address this important question (53).

Chondrocytes rarely replicate in cartilage homeostasis (54), suggesting that senescence responses other than replicative senescence might be responsible for the presence of SnCs in OA joints. Whatever the case, SnCs nonetheless secrete SASP factors, including several inflammatory mediators and enzymes capable of digesting ECM (55). Thus, the senescent chondrocyte SASP can cause an imbalance between cartilage synthesis and removal, resulting in structural dysfunction. This process might be driven by oxidative stress, mitochondrial dysfunction, genomic or epigenomic damage, or other senescence-inducing stressors (20, 56-58).

Oxidative stress induces chondrocyte senescence, primarily through upregulating p53 and p21 expression (48) and activating p38 MAPK and PI3K/Akt signaling pathways (59). This in turn stimulates a SASP (60). In addition, ROS can damage telomeres independent of replication (51), resulting in senescence and matrix loss, as well as chondrocyte apoptosis. This can produce senescence and a SASP that is independent of replication as well as chondrocyte apoptosis $(61,62)$. In chondrocytes, ROS-induced mitochondrial dysfunction can amplify chondrocyte inflammatory and matrix catabolic responses to IL- $1 \beta$ and TNF, mediated by NF- $\kappa \mathrm{B}$ activation $(61,62)$. Further, owing to the mechanoresponsive nature of chondrocytes, excessive loading of articular surfaces caused by acute joint trauma or posttraumatic joint instability can cause a release of ROS and increased oxidative stress in chondrocytes, which can also induce senescence (21). High shear stress alone can induce chondrocyte senescence $(18,21)$. In our murine studies, ACL transection (ACLT) induced senescence in the superficial layer or chondrocytes in young animals, while SnCs were found throughout the cartilage in aged animals (22).

Subchondral bone. Cartilage and the adjacent subchondral bone are connected mechanically, physically, and biologically. Both have been implicated in disease initiation and progression (63). Subchondral bone sclerosis is a hallmark of OA progression. It is still controversial whether bone changes in OA joints are a cause or a consequence of articular cartilage dysfunction and destruction (64-66). Loss of subchondral bone volume (67) and thinning of the subchondral bone plate $(68,69)$, originating from increased bone remodeling $(70,71)$, are early signs of disease in both surgically induced and spontaneous OA models. In late-stage OA, subchondral bone becomes sclerotic and stiffens, owing to decreased bone resorption. This biphasic change in subchondral bone properties is found in both preclinical animal models and human disease (72, 73). TGF- $\beta$ activity is present in the subchondral bone at the onset of ACLT-induced PTOA. This is followed by migration and formation of osteoprogenitors, subchondral bone sclerosis, and proteoglycan loss in articular cartilage tissue (74). Despite preclinical validation of the protective effects of bone-targeting drugs (e.g., bisphosphonate, strontium ranelate, cathepsin $\mathrm{K}$ inhibitor, and TGF- $\beta$ type I receptor inhibitor [SB-505124] and TGF- $\beta$ antibody [1D11]) on both subchondral bone and cartilage erosion, targeted bone in OA remains to be validated in the clinic (74-77).

In the ACLT-induced OA model, we found that $\mathrm{p} 16^{\mathrm{INK} 4 \mathrm{~A}}-$ positive SnCs were present in the subchondral BM of aged mice (19-20 months old) (22). The evolution and impact of SnC burden in subchondral bone during OA progression in both human and experimentally induced animal models are not yet defined. Studies on the central core of long bones and BM provide some insight into SnCs in this tissue (78-80). In old animals, senescence was found in osteocytes and myeloid cells retrieved from both trabecular and cortical bone tissue. Moreover, these cells' SASP was implicated in agerelated bone loss (80). Thus, SnC burden in bone can have a significant physiological impact and may produce the subchondral bone changes and bone remodeling that are relevant to OA progression. 
Infrapatellar fat pad. Adipose tissue is rich in inflammatory cells. The increased incidence of OA in obese individuals may be due to both increased mechanical load and biological factors associated with metabolic disease. Accumulating evidence points to IPFPs, located inside and near the joint capsule, as another potential source of proinflammatory cytokines and chemokines in OA. Adipokines such as leptin and adiponectin are found in human diseased synovial fluid and animal models of OA (81-83). Like other adipose depots, IPFPs contain immune cells such as macrophages and leukocytes that can respond to and/or secrete inflammatory cytokines, including SASP-associated TNF and IL-6 (84). IPFPs may contribute to inflammation in the joint, either in response to SnCs in other joint tissues or owing to their own harboring of SnCs (22). Considering the impact of obesity on OA development, further investigation into SnCs in IPFPs is warranted.

Synovium. The synovium's role in OA has long been postulated, but it remains unclear whether the changes in the synovium are primary or occur secondary to joint inflammation and cartilage breakdown (85-87). The synovium is infiltrated by immune cells during disease (86). During joint inflammation there are synovial hyperplasia and increased cell number, termed synovitis. Macrophage infiltration and production of inflammatory mediators, including IL-1 $\beta$, TNF, VEGF, and intercellular adhesion molecules (e.g., VCAM1 and E-selectin), increase in patients in the early phases of OA compared with late-phase OA (85). Synovial macrophages also contribute to osteophyte formation and other OA-related pathology such as fibrosis via production of growth factors including BMPs and TGF- $\beta$ after joint injury (88, 89). T cell activation and production of Th1 cytokines (IFN- $\gamma$, IL-2, and -10) also occurs in the arthritic synovium, implicating the initiation or amplification of inflammation by immune cells within the synovium and involvement of the adaptive immune system during OA progression (90).

There are multiple possible connections between the synovium, SnCs, and OA. SnCs in other joint tissues can impact the synovium through their SASPs, whose many chemokines and cytokines can attract and modulate resident and migrating immune cells in the synovium. In addition, synovial fibroblasts secrete less synovial fluid, which is rich in hyaluronan and lubricin (also called superficial zone protein), leading to increased cartilage degradation (91). The fibroblasts also upregulate catabolic genes (e.g., MMP-1 and -13 and the aggrecanases ADAMTS-4 and -5) and proinflammatory cytokines (e.g., IL-1, IL-6, TNF- $\alpha$, NO, and prostaglandin $\left.\mathrm{E}_{2}\left[\mathrm{PGE}_{2}\right]\right)$ that contribute to joint destruction and OA pain $(85,92)$. As cells in the synovium become proliferative and activated (93), they may themselves become more susceptible to undergoing senescence. We found that p16 ${ }^{\mathrm{INK} 4 \mathrm{~A}}$-positive SnCs were present in the synovium in the ACLT-induced OA model (22).

\section{Senescence and OA: is there causation?}

SnCs are present in most, if not all, tissues of the arthritic joint. Until recently, it was not clear whether SnCs are responsible for disease development and, more importantly, whether their removal would provide a therapeutic benefit. Recent studies provide some insight into the role of SnCs in OA development.

Lessons from transgenic models. Senescent chondrocytes are associated with both posttraumatic and age-related OA, yet their role in disease causation is unknown. To fill this gap in our knowledge, we used the p16-3MR transgenic mouse model, which enables both visualization and selective elimination (senolysis) of p16 ${ }^{\text {INK4A }}$-expressing cells at any point in the lifespan, accomplished by 16 promoter-driven expression of the herpes simplex virus thymidine kinase and administration of an otherwise benign drug, ganciclovir (GCV) $(22,35,94)$.

We found that SnCs increased in the joint over a 2-week period and then decreased to a steady-state level that remained above baseline after PTOA-inducing ACLT surgery. Expression of the SASP factors MMP-13, IL-6, and IL-1 $\beta$ evoked similar changes in the joint space. Intra-articular GCV injections selectively eliminated SnCs in the cartilage and synovium (not IPFP), leading to reduced SASP expression and pain in the surgically treated limb and cartilage tissue growth in young animals. Ultimately, eliminating SnCs and their SASP enabled tissue recovery in young animals. Additional work that induced an OA-like phenotype by injection of SnCs from ear cartilage into the knee joint region of mice could partially explain this observation (95). However, new tissue development did not occur after senolysis in aged animals (22), possibly because of irreversible damage that occurs during aging or other systemic factors involved in tissue repair processes.

While OA occurs in younger patients who experience injury, age-associated $\mathrm{OA}$ is a prevalent condition with substantial clinical and economic burden. The impact of $\mathrm{SnC}$ clearance on age-related OA was tested in another transgenic mouse model (INK-ATTAC) that allows selective killing of SnCs using a drug that promotes caspase-dependent apoptosis (44). The articular cartilage of animals whose SnCs were systemically removed starting at 12 months of age until natural death had a structural and matrix appearance similar to that of young healthy animals. Because treatment started at 12 months of age, it is likely that some level of OA was present when treatment was initiated. The results, then, suggest that, like PTOA, SnC clearance not only prevented disease progression but restored tissue structure. This complete reduction in age-related cartilage loss was observed in females, whereas our PTOA studies were performed in male mice. Further studies are needed to determine the effect of sexual phenotype on the efficacy and impact of SnC clearance for OA treatment.

Relevance to human disease and drug development. Preclinical models, particularly transgenic mice, are useful tools to define disease pathogenesis and drug mechanisms of action but may not fully reflect human disease. OA presents a unique opportunity to establish human relevance through access to tissue from patients undergoing total knee arthroplasty (TKA). Chondrocytes can be isolated and cultured from TKA cartilage tissue. While patient variability exists, in our experience and in the literature, a consistent percentage of senescent chondrocytes are present in OA patient-derived chondrocytes $(21,46,49,50)$. We found that treating these chondrocytes with a small-molecule senolytic reduced $\mathrm{SnC}$ burden and upregulated expression and deposition of type II collagen and aggrecan, the two primary ECM components of cartilage (22). Upregulation of matrix synthesis was also observed in $16^{\mathrm{INK} 4 \mathrm{~A}}$-silenced human OA chondrocytes, although this may not be relevant to the in vivo scenario (50). With so few options for isolating $\mathrm{SnCs}$ from tissues, the articular joint provides a unique source for obtaining human cells. 


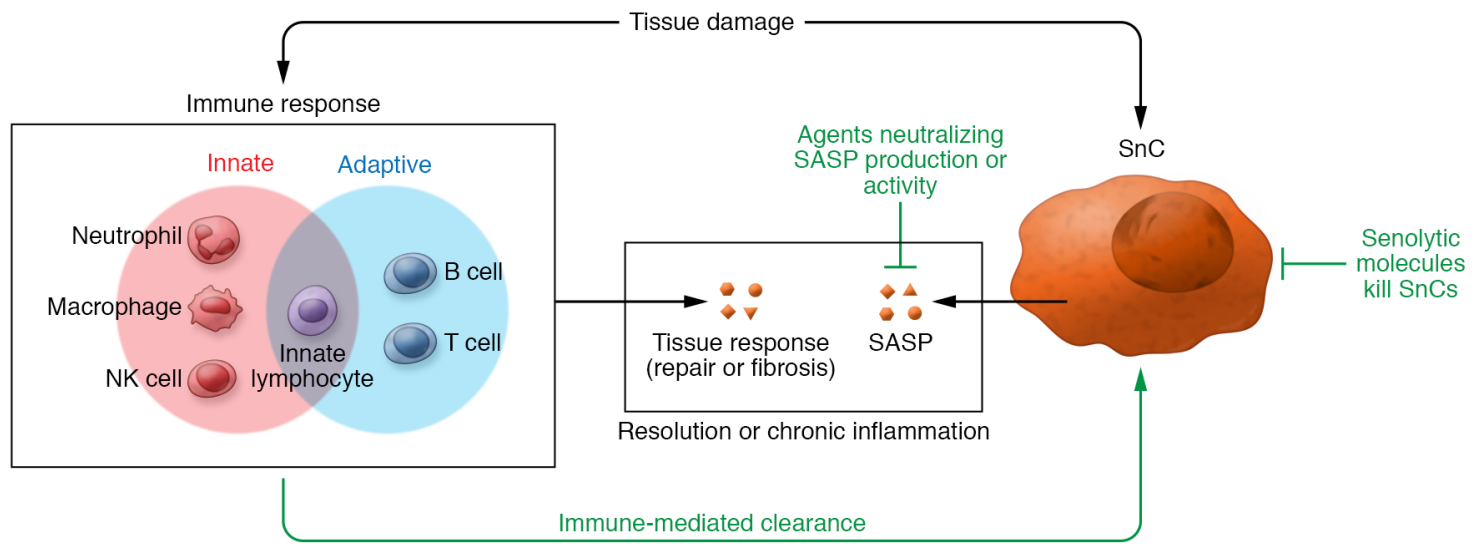

Figure 2. Senescent cells develop a SASP. The nature of the SASP depends on the local microenvironment and will itself alter the local environment. The innate and adaptive immune system can respond to joint trauma and tissue injury. The SASP includes cytokines that attract and modulate immune cells, which remove debris and secrete cytokines that influence the tissue response toward repair or fibrosis. Elements of the immune system are also responsible for clearing SnCs that form after injury. When persistent, the molecules secreted by both SnCs and immune cells cause chronic inflammation and ultimately chronic disease. Three strategies (green text) potentially prevent or attenuate OA diseases by selectively eliminating SnCs: augmentation of immune-mediated SnC clearance (e.g., chimeric antigen receptor [CAR] T cells, which are engineered to express immunoglobulin variable fragments to SnC surface markers), SASP neutralization, and direct killing of SnCs by senolytics.

\section{Senescence, pain, and inflammation}

Joint pain is the primary factor that brings patients into the clinic. While pain does not always correlate with tissue degradation in the joint, it is a critical feature of OA. Biological mechanisms of joint pain remain unclear, but local inflammation is a likely cause (96). Elevated levels of TNF- $\alpha$, a SASP factor, correlate with pain in OA joints. Supporting the relevance of SnCs to pain, we found that pain was relieved in the joints soon after SnC clearance, before any tissue structural changes were possible (22). The synovium is a likely contributor of pain-inducing cytokines, but other cell types may also be responsible. Immune cells, present in multiple tissues in the joint, also secrete cytokines that can induce pain. Understanding SASP factors that cause pain, whether secreted by synovial cells, immune cells, or other cells in joint tissues, will improve understanding of the clinical features of $\mathrm{OA}$ and potential therapeutic efficacy of senolytics (97).

Joint trauma and inflammation. The immune system is the guardian of tissue integrity (98). SnCs and their SASP are a link to the immune system and therefore the tissue damage response. Features of the immune response to tissue damage determine outcomes of repair (Figure 2). After trauma, the immune system is a first responder to the injury site, clearing debris and actively participating in tissue remodeling, which is critical to the repair process. Immune cells secrete cytokines that stimulate tissue rebuilding, including stem cell differentiation, vascular development, resident tissue activation, and ECM synthesis. Whether initiated by trauma or infection, the local tissue environment dictates immune responses (98). Thus, both the tissue-specific SASPs and the resulting tissue-specific immune response likely work together to define subsequent tissue repair, fibrosis, or chronic disease pathogenesis.

Joint trauma initiates an immune response by sending danger signals, including DAMPs. In OA, DAMPs that increase include alarmins such as HMGB1 and products of cartilage breakdown such as hyaluronan, keratan sulfate, and cartilage oligomeric matrix protein. These signals are first received by the innate immune system (98). The DAMPs released into the synovial fluid generate proinflammatory and catabolic mediators by signaling through pattern recognition receptors on OA chondrocytes and synovial cells, including TLRs and receptor for advanced glycation end-products (RAGE). Specific to OA, TLR1-TLR7 and TLR9 are activated in the synovium of patients with OA. Through these receptors, MyD88-dependent signaling activates NF- $\mathrm{KB}$, the proinflammatory stress-related transcription factor, followed by secretion of SASP-affiliated cytokines (IL-1, IL-6, IL-15, and TNF- $\alpha$ ) and chemokines (IL-8, CCL5, and CCL19) $(86,99)$.

DAMPs activate the early innate complement proteolytic cascade through the leakage of plasma complement proteins into the articular joint. Complement components and inhibitors (e.g., the central component effectors $\mathrm{C} 3 \mathrm{a}$ and $\mathrm{C} 5 \mathrm{a}$ ) are increased in synovial macrophages and chondrocytes in OA (100). Indeed, complement component deficiency protects animals from surgically induced and spontaneous OA development (101).

Macrophages are the innate immune cells that initially migrate to a wound. Macrophages can acquire proinflammatory, proregenerative, or profibrotic phenotypes. Proinflammatory macrophage phenotypes promote phagocytosis and debris removal in damaged tissue by producing TNF- $\alpha$, IL-1, and IL- 6 . In normal wound healing, proinflammatory macrophages transition to proregenerative or profibrotic phenotypes to promote tissue replacement in response to IL-4, IL-10, and IL-13 or other immune complexes. Macrophages are a heterogeneous cell population, and the impact of their phenotype on OA development remains unknown.

The role of the adaptive immune system is recognized in inflammatory arthritis, but there is still limited evidence to date on the adaptive immune system's role in OA. However, ongoing studies on the changes in adaptive immune cells in trauma and aging-related OA will address this role. Generally, adaptive responses activate resident or infiltrating macrophages as well as $\mathrm{T}$ and B lymphocytes $(91,99)$, which orchestrate many aspects of tissue repair, including development of myeloid phenotypes. In 
muscle repair, IL-4 production by Th2 cells was required for the myeloid cell transition to a proregenerative phenotype, needed for efficient muscle repair (102). In the joint, IL-4 production correlated with tissue repair after ACLT, suggesting that similar mechanisms of type 2 immune responses impact OA (103). Without treatment, OA signatures reflect a profibrotic and proinflammatory immune response that is potentially mediated by SnCs, which may only recruit and modulate the phenotypes of immune cells.

SnCs and the immune system. SnCs develop early in the joint after ACLT injury and secrete cytokines that can attract and modulate immune cells. Thus, SnCs can serve as sentinels, activating and calling immune cells to the injury site to initiate repair. During normal healing, the immune system clears SnCs. In skin wounds, for example, clearing SnCs too early in the repair process delays wound healing (35). The tissue environment's impact on SnCs and the SASP likely influences a tissue-specific immune response to injury. Matzinger pioneered the concept of tissue-based immune cell effector class control (98). She postulated that the local tissue environment determined the immune response to foreign invaders, directing the type and degree of immune cell stimulation (98). This idea can explain why tissues like skin and liver have a strong capacity for repair after damage, whereas articular cartilage has limited repair capacity. The concept of tissue-specific immune cell class control likely also applies to immune responses to joint trauma and resulting wound healing responses, including the immune clearance of SnCs.

Understanding of how the immune system interacts with and ultimately clears SnCs is in its infancy. SnCs appear to attract an innate immune response that, in part, results in their elimination (104). NK cells recognize stressed or damaged cells via cell surface receptors and ligands. Specifically, some SnCs express ligands for NK cells, including those that bind NK cell receptors (e.g., NK group 2D [NKG2D] and nonclassical MHC I molecules such as HLA-E) (105-109). In liver, NK cells target senescent stellate cells using perforin granule exocytosis (110). Senescent stellate cells secrete factors that favor macrophage polarization toward a proinflammatory $\mathrm{M} 1$ state, which also promotes $\mathrm{SnC}$ clearance (111). Age-related senescence in BM may influence immune system homeostasis and the ability to clear SnCs. Systemic senolytic treatment in aged animals restores BM populations (94). While BM stem cells were a primary focus of this study, results suggest the possibility of improving age-related immune declines that can impact $\mathrm{SnC}$ clearance and tissue repair.

Macrophages can be critical players in clearing and are also required for tissue repair and regeneration $(112,113)$. Senescent stromal cells can attract or induce a subset of macrophages that reversibly express certain senescence markers, suggesting that some cells positive for $\mathrm{p} 16^{\mathrm{INK} 4 \mathrm{~A}}$ and senescence-associated $\beta$-galactosidase (SA $\beta$-gal) in aging and diseased tissues may be macrophages (114). These macrophage phenotypes, which appear to be part of the non-cell-autonomous effects of SnCs, are stimulated by IL- 4 and thus may be proregenerative.

Certain SnC-secreted SASP molecules and immune cellsecreted cytokines are known to cause pain and may be implicated in OA clinical symptoms. Examples include proinflammatory mediators from the interleukin family (IL-1 and IL-6), TNF- $\alpha$, and arachidonate-derived $\mathrm{PGE}_{2} / \mathrm{COX}-2$. Among these, $\mathrm{PGE}_{2}$ is con- sidered the major contributor to exaggerated pain sensations via E prostanoid receptors (EP1-4), present in peripheral sensory neurons that process potentially damaging stimuli and send signals through the spinal cord to the brain, usually causing the perception of pain (115). In human OA explants, $\mathrm{PGE}_{2}$ and COX-2 were elevated in the synovium, bone, and meniscus $(116,117)$. In our murine PTOA model, senolytic treatment reduced pain in a short time, before any tissue structural changes could have occurred (on day 14 after intra-articular injection of GCV, starting 8 days after ACLT surgery) (22). Thus, SnCs and their related immune stimulation may be responsible for both clinical symptoms and underlying pathogenesis of $\mathrm{OA}$.

\section{Implications of SnCs for OA treatment strategies} The causative role of SnCs and the SASP in OA opens the door to new strategies for treatment. Current pharmacological OA treatment options are limited to symptomatic relief, but none of them in fact can halt or even reverse the disease in the patients. Analgesics, NSAIDs, and intra-articular viscosupplementation (e.g., hyaluronic acid) achieve short-term symptomatic pain relief and care of joint function (3). Other therapeutic strategies for OA include intra-articular platelet-rich plasma injections, and an autologous undefined mixture of bioactive molecules purported to improve wound healing (118). Lubricin, hyaluronic acid, and biomimetic aggrecan are biophysical strategies designed to reduce cartilage deterioration (119), but their efficacy varies, demonstrating either no marked difference in effect, or only small, short-term benefits (120). With advances in the understanding of OA pathogenesis, treatment strategies are moving beyond symptomatic relief to disease-modifying OA drugs, for example, by targeting specific catabolic signaling pathways $(55,121)$.

SASP inhibition. Even before a causative role for SnCs in OA was suggested, treatment strategies were moving toward targeting molecules that are also SASP factors. These targets include proinflammatory cytokines, chemokines, growth factors, MMP13 , and ADAMTS, implicated in cartilage degradation. They also include signaling cascades upstream of the SASP, such as NF- $\mathrm{BB}$ or p38 MAPK. Targeting of inflammatory pathways is a growing area of development, particularly for inflammatory arthritis. Inhibitors of IL-1 $\beta$, TNF- $\alpha$, and IL- 6 are approved for treating inflammatory arthritis but have not been applied to OA. The clinical studies of an MMP-13 inhibitor (PG-116800) (122) and IL-1 receptor antagonists (kineret, orthokin, and AMG 108) (123-125) for OA treatment were terminated without clear benefit. Importantly, p16 $6^{\text {INK4A }}$-positive cells that secrete DAMPs may not always express a SASP $(35,41)$. While targeting SASP factors may reduce clinical symptoms of inflammation and pain, it does not address the underlying source of inflammation and tissue degradation. Continual treatment to combat SASP factors would be required if the underlying source is not eliminated.

Directly targeting SnCs to treat OA. Direct targeting and killing of SnCs provides a potential opportunity to eliminate the source of OA disease. Several senolytics have been identified, and current research continues to develop new and/or more selective drugs. Senolysis has been achieved by inhibition of antiapoptotic proteins in SnCs, including the BCL-2 family members BCL-2, BCL-XL, and BCL-W $(94,126)$. For example, the senolytic mol- 
ecule ABT-263 occupies the inhibitory binding grooves of these proteins, counteracting their antiapoptotic functions and permitting SnCs to initiate apoptosis. Treatment of sublethally irradiated mice with ABT-263 reduced the SnC burden in BM and partially restored hematopoietic function (94).

Small molecules that inhibit antiapoptotic pathways eliminated SnCs that developed after joint trauma and those present in cultured human OA chondrocytes (22). Other approaches to eliminate SnCs may also be efficacious for arthritis treatment. For example, SnCs increase expression of the transcriptional regulator FOXO4, which binds p53 and prevents p53-mediated apoptosis; a peptide that interferes with the FOXO4-p53 interaction was recently shown to induce apoptosis in SnCs $(127,128)$. Another example of a selective SnC killing strategy is inhibition of HSP90, a ubiquitously expressed molecular chaperone that is upregulated in cancer cells, where it stabilizes antiapoptotic factors (129). HSP9O inhibitors (e.g., geldanamycin and tanespimycin) induced apoptosis of SnCs by multiple mechanisms (129).

Directly targeting mechanisms that induce SnCs in OA, and cartilage in particular, may also lead to new therapeutic targets, although there is always a danger of promoting cancer in such strategies. SIRT6 depletion induced senescence in human chondrocytes, suggesting that upregulation of this protein deacetylase might suppress senescence and the SASP (130). Further studies on SIRT6's role in the human chondrocyte may help elucidate the pathogenesis of OA and confirm the potential of SIRT6 as a therapeutic target.

\section{Challenges and conclusions}

Although the targeting and removal of SnCs may provide opportunities for disease modification rather than symptomatic treatment, it is still difficult to make conclusions about the causative role of
SnCs in arthritic disease. Almost all previous publications were not carefully controlled in terms of the number of markers of senescence (SA $\beta$-gal staining was solely used) or selection of patients, who mostly had clinically advanced disease. Future research needs to define biomarkers of senescence in patients with mild, moderate, and severe OA. The presence of SnCs and impact of senolytic treatment should also be tested in other trauma-induced arthritis models such as the DMM mouse or the medial meniscus transection (MMT) rat. Moreover, there are likely significant SASP differences between trauma-induced and aging-induced SnCs. These differences in SnC phenotypes and local environmental cues may impact which drugs are required for SnC clearance. Thus, tissuespecific SASP profiles should be studied to elucidate detailed mechanistic roles of SnCs in the various joint structures. Finally, understanding the link between SnCs, the immune response to trauma, and its known deficiency in aging will likely uncover disease mechanisms and additional therapeutic targets.

\section{Acknowledgments}

The authors gratefully acknowledge financial support from Unity Biotechnology, the Morton Goldberg Chair, the Bloomberg Kimmel Institute for Cancer Immunotherapy, the NIH (AG009909), and the Office of the Assistant Secretary of Defense for Health Affairs through the Peer Reviewed Medical Research Program (W81XWH-17-1-0627).

Address correspondence to: Jennifer H. Elisseeff, Translational Tissue Engineering Center, Wilmer Eye Institute and Department of Biomedical Engineering, Johns Hopkins University, Smith Building, Room 5035, 400 N. Broadway, Baltimore, Maryland 21231, USA. Phone: 410.614.6837; Email: jhe@jhu.edu.
1. Barbour KE, Helmick CG, Boring M, Brady TJ. Vital signs: prevalence of doctor-diagnosed arthritis and arthritis-attributable activity limitation - United States, 2013-2015. MMWR Morb Mortal Wkly Rep. 2017;66(9):246-253.

2. Neogi T, Zhang Y. Epidemiology of osteoarthritis. Rheum Dis Clin North Am. 2013;39(1):1-19.

3. Wieland HA, Michaelis M, Kirschbaum BJ, Rudolphi KA. Osteoarthritis - an untreatable disease? Nat Rev Drug Discov. 2005;4(4):331-344.

4. van der Kraan PM, van den Berg WB. Osteophytes: relevance and biology. Osteoarthr Cartil. 2007;15(3):237-244.

5. Martel-Pelletier J, et al. Osteoarthritis. Nat Rev Dis Primers. 2016;2:16072.

6. Howell R, Kumar NS, Patel N, Tom J. Degenerative meniscus: pathogenesis, diagnosis, and treatment options. World J Orthop. 2014;5(5):597-602.

7. Showery JE, Kusnezov NA, Dunn JC, Bader JO, Belmont PJ Jr, Waterman BR. The rising incidence of degenerative and posttraumatic osteoarthritis of the knee in the United States military. JArthroplasty. 2016;31(10):2108-2114.

8. Sommerlath K, Lysholm J, Gillquist J. The longterm course after treatment of acute anterior cruciate ligament ruptures. A 9 to 16 year followup. Am J Sports Med. 1991;19(2):156-162.

9. Jones G, Ding C, Glisson M, Hynes K, Ma D,
Cicuttini F. Knee articular cartilage development in children: a longitudinal study of the effect of sex, growth, body composition, and physical activity. Pediatr Res. 2003;54(2):230-236.

10. Roos H, Adalberth T, Dahlberg L, Lohmander LS. Osteoarthritis of the knee after injury to the anterior cruciate ligament or meniscus: the influence of time and age. Osteoarthritis Cartilage. 1995;3(4):261-267.

11. Goldring MB, Marcu KB. Cartilage homeostasis in health and rheumatic diseases. Arthritis Res Ther. 2009;11(3):224.

12. Kapoor M, Martel-Pelletier J, Lajeunesse D, Pelletier JP, Fahmi H. Role of proinflammatory cytokines in the pathophysiology of osteoarthritis. Nat Rev Rheumatol. 2011;7(1):33-42.

13. Wojdasiewicz P, Poniatowski ŁA, Szukiewicz D. The role of inflammatory and anti-inflammatory cytokines in the pathogenesis of osteoarthritis. Mediators Inflamm. 2014;2014:561459.

14. Chang JC, et al. Global molecular changes in a tibial compression induced ACL rupture model of post-traumatic osteoarthritis. JOrthop Res. 2017;35(3):474-485.

15. Dunn SL, Soul J, Anand S, Schwartz JM, Boot-Handford RP, Hardingham TE. Gene expression changes in damaged osteoarthritic cartilage identify a signature of non-chondrogenic and mechanical responses. Osteoarthr Cartil.
2016;24(8):1431-1440.

16. Steinberg J, et al. Integrative epigenomics, transcriptomics and proteomics of patient chondrocytes reveal genes and pathways involved in osteoarthritis. Sci Rep. 2017;7(1):8935.

17. Barenius B, Ponzer S, Shalabi A, Bujak R, Norlén L, Eriksson K. Increased risk of osteoarthritis after anterior cruciate ligament reconstruction: a 14-year follow-up study of a randomized controlled trial. Am J Sports Med. 2014;42(5):1049-1057.

18. Martin JA, Brown TD, Heiner AD, Buckwalter JA. Chondrocyte senescence, joint loading and osteoarthritis. Clin Orthop Relat Res. 2004; (427 Suppl):S96-S103.

19. Childs BG, Durik M, Baker DJ, van Deursen JM. Cellular senescence in aging and age-related disease: from mechanisms to therapy. Nat Med. 2015;21(12):1424-1435.

20. Loeser RF. Aging and osteoarthritis: the role of chondrocyte senescence and aging changes in the cartilage matrix. Osteoarthr Cartil. 2009;17(8):971-979.

21. Martin JA, Brown T, Heiner A, Buckwalter JA. Post-traumatic osteoarthritis: the role of accelerated chondrocyte senescence. Biorheology. 2004;41(3-4):479-491.

22. Jeon $\mathrm{OH}$, et al. Local clearance of senescent cells attenuates the development of post-traumatic osteoarthritis and creates a pro-regenerative 
environment. Nat Med. 2017;23(6):775-781.

23. Lopez-Otin C, Blasco MA, Partridge L, Serrano M, Kroemer G. The hallmarks of aging. Cell. 2013;153(6):1194-1217.

24. Campisi J. Aging, cellular senescence, and cancer. Annu Rev Physiol. 2013;75:685-705.

25. Titus S, et al. Impairment of BRCA1-related DNA double-strand break repair leads to ovarian aging in mice and humans. Sci Transl Med. 2013;5(172):172ra21.

26. Booth LN, Brunet A. The aging epigenome. Mol Cell. 2016;62(5):728-744.

27. Serrano M, Lin AW, McCurrach ME, Beach D, Lowe SW. Oncogenic ras provokes premature cell senescence associated with accumulation of p53 and p16INK4a. Cell. 1997;88(5):593-602.

28. Ksiazek K, Mikula-Pietrasik J, Olijslagers S, Jörres A, von Zglinicki T, Witowski J. Vulnerability to oxidative stress and different patterns of senescence in human peritoneal mesothelial cell strains. Am J Physiol Regul Integr Comp Physiol. 2009;296(2):R374-R382.

29. Wiley CD, et al. Mitochondrial dysfunction induces senescence with a distinct secretory phenotype. Cell Metab. 2016;23(2):303-314.

30. Campisi J, d'Adda di Fagagna F. Cellular senescence: when bad things happen to good cells. Nat Rev Mol Cell Biol. 2007;8(9):729-740.

31. Childs BG, Durik M, Baker DJ, van Deursen JM. Cellular senescence in aging and age-related disease: from mechanisms to therapy. Nat Med. 2015;21(12):1424-1435.

32. Alimonti A, et al. A novel type of cellular senescence that can be enhanced in mouse models and human tumor xenografts to suppress prostate tumorigenesis. J Clin Invest. 2010;120(3):681-693.

33. Muñoz-Espín D, et al. Programmed cell senescence during mammalian embryonic development. Cell. 2013;155(5):1104-1118.

34. Storer M, et al. Senescence is a developmental mechanism that contributes to embryonic growth and patterning. Cell. 2013;155(5):1119-1130.

35. Demaria M, et al. An essential role for senescent cells in optimal wound healing through secretion of PDGF-AA. Dev Cell. 2014;31(6):722-733.

36. Helman A, et al. p16(Ink4a)-induced senescence of pancreatic beta cells enhances insulin secretion. Nat Med. 2016;22(4):412-420.

37. Campisi J. Cancer, aging and cellular senescence. In Vivo. 2000;14(1):183-188.

38. van Deursen JM. The role of senescent cells in ageing. Nature. 2014;509(7501):439-446.

39. Coppé JP, et al. Senescence-associated secretory phenotypes reveal cell-nonautonomous functions of oncogenic RAS and the p53 tumor suppressor. PLoS Biol. 2008;6(12):2853-2868.

40. Orjalo AV, Bhaumik D, Gengler BK, Scott GK, Campisi J. Cell surface-bound IL-1alpha is an upstream regulator of the senescence-associated IL-6/IL-8 cytokine network. Proc Natl Acad Sci US A. 2009;106(40):17031-17036.

41. Coppé JP, Rodier F, Patil CK, Freund A, Desprez PY, Campisi J. Tumor suppressor and aging biomarker p16(INK4a) induces cellular senescence without the associated inflammatory secretory phenotype. JBiol Chem. 2011;286(42):36396-36403.

42. Davalos AR, et al. p53-dependent release of Alarmin HMGB1 is a central mediator of senes- cent phenotypes. JCell Biol. 2013;201(4):613-629.

43. Acosta JC, et al. A complex secretory program orchestrated by the inflammasome controls paracrine senescence. Nat Cell Biol. 2013;15(8):978-990.

44. Baker DJ, et al. Naturally occurring p16(Ink4a)-positive cells shorten healthy lifespan. Nature. 2016;530(7589):184-189.

45. Philipot D, et al. p16INK4a and its regulator miR24 link senescence and chondrocyte terminal differentiation-associated matrix remodeling in osteoarthritis. Arthritis Res Ther. 2014;16(1):R58.

46. Price JS, et al. The role of chondrocyte senescence in osteoarthritis. Aging Cell. 2002;1(1):57-65.

47. Sandell LJ. Etiology of osteoarthritis: genetics and synovial joint development. Nat Rev Rheumatol. 2012;8(2):77-89.

48. Dai SM, et al. Catabolic stress induces features of chondrocyte senescence through overexpression of caveolin 1: possible involvement of caveolin 1-induced down-regulation of articular chondrocytes in the pathogenesis of osteoarthritis. Arthritis Rheum. 2006;54(3):818-831.

49. Gao SG, et al. Correlation between senescence-associated beta-galactosidase expression in articular cartilage and disease severity of patients with knee osteoarthritis. Int J Rheum Dis. 2016;19(3):226-232.

50. Zhou HW, Lou SQ, Zhang K. Recovery of function in osteoarthritic chondrocytes induced by p16INK4a-specific siRNA in vitro. Rheumatology (Oxford). 2004;43(5):555-568.

51. Martin JA, Klingelhutz AJ, Moussavi-Harami F, Buckwalter JA. Effects of oxidative damage and telomerase activity on human articular cartilage chondrocyte senescence. J Gerontol A Biol Sci Med Sci. 2004;59(4):324-337.

52. Martin JA, Buckwalter JA. Human chondrocyte senescence and osteoarthritis. Biorheology. 2002;39(1-2):145-152.

53. Unity Biotechnology Inc. An Exploratory Clinical Study to Investigate Biomarkers of Senescence in Patients With Osteoarthritis of the Knee. NIH Website. https://clinicaltrials.gov/ct2/show/ NCT03100799. Accessed February 15, 2018.

54. Goldring MB, Goldring SR. Osteoarthritis. JCell Physiol. 2007;213(3):626-634.

55. Mobasheri A. The future of osteoarthritis therapeutics: targeted pharmacological therapy. Curr Rheumatol Rep. 2013;15(10):364.

56. Koike M, et al. Mechanical overloading causes mitochondrial superoxide and SOD2 imbalance in chondrocytes resulting in cartilage degeneration. Sci Rep. 2015;5:11722.

57. Rose J, et al. DNA damage, discoordinated gene expression and cellular senescence in osteoarthritic chondrocytes. Osteoarthr Cartil. 2012;20(9):1020-1028.

58. Harbo M, et al. The distribution pattern of critically short telomeres in human osteoarthritic knees. Arthritis Res Ther. 2012;14(1):R12.

59. Yu SM, Kim SJ. Thymoquinone-induced reactive oxygen species causes apoptosis of chondrocytes via PI3K/Akt and p38kinase pathway. Exp Biol Med (Maywood). 2013;238(7):811-820.

60. Freund A, Patil CK, Campisi J. p38MAPK is a novel DNA damage response-independent regulator of the senescence-associated secretory phenotype. EMBO J. 2011;30(8):1536-1548.

61. Vaamonde-García C, Riveiro-Naveira RR, Valcárcel-Ares MN, Hermida-Carballo L, Blanco FJ, López-Armada MJ. Mitochondrial dysfunction increases inflammatory responsiveness to cytokines in normal human chondrocytes. Arthritis Rheum. 2012;64(9):2927-2936.

62. Gavriilidis C, Miwa S, von Zglinicki T, Taylor RW, Young DA. Mitochondrial dysfunction in osteoarthritis is associated with down-regulation of superoxide dismutase 2. Arthritis Rheum. 2013;65(2):378-387.

63. Burr DB, Gallant MA. Bone remodelling in osteoarthritis. Nat Rev Rheumatol. 2012;8(11):665-673.

64. Radin EL, Paul IL, Tolkoff MJ. Subchondral bone changes in patients with early degenerative joint disease. Arthritis Rheum. 1970;13(4):400-405.

65. Radin EL, Martin RB, Burr DB, Caterson B, Boyd RD, Goodwin C. Effects of mechanical loading on the tissues of the rabbit knee. JOrthop Res. 1984;2(3):221-234.

66. Carlson CS, Loeser RF, Purser CB, Gardin JF, Jerome CP. Osteoarthritis in cynomolgus macaques. III: Effects of age, gender, and subchondral bone thickness on the severity of disease. J Bone Miner Res. 1996;11(9):1209-1217.

67. Hayami T, Pickarski M, Zhuo Y, Wesolowski GA, Rodan GA, Duong LT. Characterization of articular cartilage and subchondral bone changes in the rat anterior cruciate ligament transection and meniscectomized models of osteoarthritis. Bone. 2006;38(2):234-243.

68. Sniekers YH, et al. A role for subchondral bone changes in the process of osteoarthritis; a microCT study of two canine models. BMC Musculoskelet Disord. 2008;9:20.

69. Intema F, et al. Similarities and discrepancies in subchondral bone structure in two differently induced canine models of osteoarthritis. JBone Miner Res. 2010;25(7):1650-1657.

70. Amir G, Pirie CJ, Rashad S, Revell PA. Remodelling of subchondral bone in osteoarthritis: a histomorphometric study. J Clin Pathol. 1992;45(11):990-992.

71. Benske J, Schünke M, Tillmann B. Subchondral bone formation in arthrosis. Polychrome labeling studies in mice. Acta Orthop Scand. 1988;59(5):536-541.

72. Hannan MT, Anderson JJ, Zhang Y, Levy D, Felson DT. Bone mineral density and knee osteoarthritis in elderly men and women. The Framingham Study. Arthritis Rheum. 1993;36(12):1671-1680.

73. Bolbos RI, et al. Relationship between trabecular bone structure and articular cartilage morphology and relaxation times in early OA of the knee joint using parallel MRI at 3 T. Osteoarthr Cartil. 2008;16(10):1150-1159.

74. Zhen $\mathrm{G}$, et al. Inhibition of TGF- $\beta$ signaling in mesenchymal stem cells of subchondral bone attenuates osteoarthritis. Nat Med. 2013;19(6):704-712.

75. Connor JR, et al. Protective effects of a cathepsin K inhibitor, SB-553484, in the canine partial medial meniscectomy model of osteoarthritis. Osteoarthr Cartil. 2009;17(9):1236-1243.

76. Reginster JY. Efficacy and safety of strontium ranelate in the treatment of knee osteoar- 
thritis: results of a double-blind randomised, placebo-controlled trial. Ann Rheum Dis. 2014;73(2):e8.

77. Carbone LD, et al. The relationship of antiresorptive drug use to structural findings and symptoms of knee osteoarthritis. Arthritis Rheum. 2004;50(11):3516-3525.

78. Burd CE, et al. Monitoring tumorigenesis and senescence in vivo with a p16(INK4a)-luciferase model. Cell. 2013;152(1-2):340-351.

79. Krishnamurthy J, et al. Ink4a/Arf expression is a biomarker of aging. J Clin Invest. 2004;114(9):1299-1307.

80. Farr JN, et al. Identification of senescent cells in the bone microenvironment. J Bone Miner Res. 2016;31(11):1920-1929.

81. Ku JH, et al. Correlation of synovial fluid leptin concentrations with the severity of osteoarthritis. Clin Rheumatol. 2009;28(12):1431-1435.

82. McNulty AL, Miller MR, O'Connor SK, Guilak F. The effects of adipokines on cartilage and meniscus catabolism. Connect Tissue Res. 2011;52(6):523-533.

83. Berenbaum F, Eymard F, Houard X. Osteoarthritis, inflammation and obesity. Curr Opin Rheumatol. 2013;25(1):114-118.

84. Ushiyama T, Chano T, Inoue K, Matsusue Y. Cytokine production in the infrapatellar fat pad: another source of cytokines in knee synovial fluids. Ann Rheum Dis. 2003;62(2):108-112.

85. Benito MJ, Veale DJ, FitzGerald O, van den Berg WB, Bresnihan B. Synovial tissue inflammation in early and late osteoarthritis. Ann Rheum Dis. 2005;64(9):1263-1267.

86. Liu-Bryan R. Synovium and the innate inflammatory network in osteoarthritis progression. Curr Rheumatol Rep. 2013;15(5):323.

87. Sellam J, Berenbaum F. The role of synovitis in pathophysiology and clinical symptoms of osteoarthritis. Nat Rev Rheumatol. 2010;6(11):625-635.

88. van Lent PL, et al. Crucial role of synovial lining macrophages in the promotion of transforming growth factor beta-mediated osteophyte formation. Arthritis Rheum. 2004;50(1):103-111.

89. Blom AB, et al. Synovial lining macrophages mediate osteophyte formation during experimental osteoarthritis. Osteoarthr Cartil. 2004;12(8):627-635.

90. Sakkas LI, Platsoucas CD. The role of T cells in the pathogenesis of osteoarthritis. Arthritis Rheum. 2007;56(2):409-424.

91. Liu-Bryan R, Terkeltaub R. Emerging regulators of the inflammatory process in osteoarthritis. Nat Rev Rheumatol. 2015;11(1):35-44.

92. Gupta A, Niger C, Buo AM, Eidelman ER, Chen RJ, Stains JP. Connexin 43 enhances the expression of osteoarthritis-associated genes in synovial fibroblasts in culture. BMC Musculoskelet Disord. 2014;15:425.

93. Smith MD. The normal synovium. Open Rheumatol J. 2011;5:100-106.

94. Chang J, et al. Clearance of senescent cells by ABT263 rejuvenates aged hematopoietic stem cells in mice. Nat Med. 2016;22(1):78-83.

95. Xu M, et al. Transplanted senescent cells induce an osteoarthritis-like condition in mice. J Gerontol A Biol Sci Med Sci. 2017;72(6):780-785.

96. Malfait AM, Schnitzer TJ. Towards a mechanismbased approach to pain management in osteoarthritis. Nat Rev Rheumatol. 2013;9(11):654-664.

97. Orita S, et al. Pain-related sensory innervation in monoiodoacetate-induced osteoarthritis in rat knees that gradually develops neuronal injury in addition to inflammatory pain. BMC Musculoskelet Disord. 2011;12:134.

98. Matzinger P, Kamala T. Tissue-based class control: the other side of tolerance. Nat Rev Immunol. 2011;11(3):221-230.

99. Scanzello CR, Goldring SR. The role of synovitis in osteoarthritis pathogenesis. Bone. 2012;51(2):249-257.

100.Bradley K, et al. Synthesis of classical pathway complement components by chondrocytes. Immunology. 1996;88(4):648-656.

101.Wang $\mathrm{Q}$, et al. Identification of a central role for complement in osteoarthritis. Nat Med. 2011;17(12):1674-1679.

102. Sadtler K, et al. Developing a pro-regenerative biomaterial scaffold microenvironment requires T helper 2 cells. Science. 2016;352(6283):366-370.

103. Jacobs HN, Rathod S, Wolf MT, Elisseeff JH. Intra-articular injection of urinary bladder matrix reduces osteoarthritis development. AAPS J. 2017;19(1):141-149.

104. Krizhanovsky V, et al. Senescence of activated stellate cells limits liver fibrosis. Cell. 2008;134(4):657-667.

105. Sagiv A, et al. NKG2D ligands mediate immunosurveillance of senescent cells. Aging (Albany NY). 2016;8(2):328-344.

106.Lackner DH, Hayashi MT, Cesare AJ, Karlseder J. A genomics approach identifies senescencespecific gene expression regulation. Aging Cell. 2014;13(5):946-950.

107. Cahu J, Bustany S, Sola B. Senescenceassociated secretory phenotype favors the emergence of cancer stem-like cells. Cell Death Dis. 2012;3:e446.

108.Sharpless NE, Sherr CJ. Forging a signature of in vivo senescence. Nat Rev Cancer. 2015;15(7):397-408.

109. Coppé JP, Desprez PY, Krtolica A, Campisi J. The senescence-associated secretory phenotype: the dark side of tumor suppression. Annu Rev Pathol. 2010;5:99-118.

110.Sagiv A, Biran A, Yon M, Simon J, Lowe SW, Krizhanovsky V. Granule exocytosis mediates immune surveillance of senescent cells. Oncogene. 2013;32(15):1971-1977.

111. Lujambio A, et al. Non-cell-autonomous tumor suppression by p53. Cell. 2013;153(2):449-460.

112. Wynn TA, Chawla A, Pollard JW. Macrophage biology in development, homeostasis and disease. Nature. 2013;496(7446):445-455.

113. Yun MH, Davaapil H, Brockes JP. Recurrent turnover of senescent cells during regeneration of a complex structure. Elife. 2015;4:e05505.

114. Hall BM, et al. p16(Ink4a) and senescenceassociated $\beta$-galactosidase can be induced in macrophages as part of a reversible response to physiological stimuli. Aging (Albany NY). 2017;9(8):1867-1884

115. Dray A, Read SJ. Arthritis and pain. Future targets to control osteoarthritis pain. Arthritis Res Ther. 2007;9(3):212.

116. Sato T, et al. Prostaglandin EP2 receptor signalling inhibits the expression of matrix metalloproteinase 13 in human osteoarthritic chondrocytes. Ann Rheum Dis. 2011;70(1):221-226.

117. Attur M, et al. Prostaglandin E2 exerts catabolic effects in osteoarthritis cartilage: evidence for signaling via the EP4 receptor. JImmunol. 2008;181(7):5082-5088.

118. Zhu Y, et al. Basic science and clinical application of platelet-rich plasma for cartilage defects and osteoarthritis: a review. Osteoarthr Cartil. 2013;21(11):1627-1637.

119. Sharma S, Vazquez-Portalatin N, Calve S, Panitch A. Biomimetic molecules lower catabolic expression and prevent chondroitin sulfate degradation in an osteoarthritic ex vivo model. ACS Biomater Sci Eng. 2016;2(2):241-250.

120.Bannuru RR, Schmid CH, Kent DM, Vaysbrot EE, Wong JB, McAlindon TE. Comparative effectiveness of pharmacologic interventions for knee osteoarthritis: a systematic review and network meta-analysis. Ann Intern Med. 2015;162(1):46-54.

121. Hunter DJ. Pharmacologic therapy for osteoarthritis - the era of disease modification. Nat Rev Rheumatol. 2011;7(1):13-22.

122. Krzeski P, et al. Development of musculoskeletal toxicity without clear benefit after administration of PG-116800, a matrix metalloproteinase inhibitor, to patients with knee osteoarthritis: a randomized, 12-month, double-blind, placebo-controlled study. Arthritis Res Ther. 2007;9(5):R109.

123. Chevalier $\mathrm{X}$, et al. Intraarticular injection of anakinra in osteoarthritis of the knee: a multicenter, randomized, double-blind, placebo-controlled study. Arthritis Rheum. 2009;61(3):344-352.

124. Auw Yang KG, et al. Autologous interleukin-1 receptor antagonist improves function and symptoms in osteoarthritis when compared to placebo in a prospective randomized controlled trial. Osteoarthr Cartil. 2008;16(4):498-505.

125. Cohen SB, et al. A randomized, double-blind study of AMG 108 (a fully human monoclonal antibody to IL-1R1) in patients with osteoarthritis of the knee. Arthritis Res Ther. 2011;13(4):R125.

126. Yosef R, et al. Directed elimination of senescent cells by inhibition of BCL-W and BCL-XL. Nat Commun. 2016;7:11190.

127. Baar MP, et al. Targeted apoptosis of senescent cells restores tissue homeostasis in response to chemotoxicity and aging. Cell. 2017;169(1):132-147.e16.

128. de Keizer PL. The fountain of youth by targeting senescent cells? Trends Mol Med. 2017;23(1):6-17.

129. Fuhrmann-Stroissnigg $\mathrm{H}$, et al. Identification of HSP9O inhibitors as a novel class of senolytics. Nat Commun. 2017;8(1):422.

130. Nagai K, et al. Depletion of SIRT6 causes cellular senescence, DNA damage, and telomere dysfunction in human chondrocytes. Osteoarthr Cartil.2015;23(8):1412-1420. 